\title{
Practice-based Assessment of Tobacco Usage in Southwestern Primary Care Patients: A Research Involving Outpatient Settings Network (RIOS Net) Study
}

\author{
Shawn Ralston, MD, Nicole Kellett, MA, Robert L. Williams, MD, MPH, \\ Cheryl Schmitt, MS, and Charles Q. North, MD, MS
}

Purpose: Primary care clinicians rely, perhaps erroneously, on general population-based data about risk factors to help form their strategies for allocating time in the brief primary care encounter. We conducted a case study using rates of tobacco usage among people presenting for primary care to explore comparability to general population-based rates.

Methods: Clinicians in RIOS Net, a practice-based research network, gathered data on tobacco use for all patients presenting during a 2 -week period. We compared those data to population-based data by gender and ethnicity.

Results: Ninety-one primary care clinicians reported data on 2442 patients. Primary care smoking rates differed in important ways from general population-based rates. Hispanic women smoked at more than twice the national population-based rate $(25 \%$ vs $12 \%)$. Youth smoked at higher rates as well, particularly young Native American men.

Conclusions: Patients seen in primary care differ in important ways in rates and patterns of tobacco usage when compared with rates reported in population-based surveys. These differences could have important implications for preventive care within the context of multiple competing demands in the primary care encounter. (J Am Board Fam Med 2007;20:174-180.)

The literature on competing demands in primary care has begun to explore the dynamics of preven-

This article was externally peer-reviewed.

Submitted 2 February 2006; revised 12 November 2006; accepted 20 November 2006.

From the Departments of Pediatrics (SR) and Family and Community Medicine (NK, RLW, CS), University of New Mexico Health Sciences Center, Albuquerque, NM; and Indian Health Service, Albuquerque Service Unit, Albuquerque, NM (CQN).

Funding: This study was supported by funding from the University of New Mexico Cancer Research and Treatment Center through grant P20CA088807-04 from the National Cancer Institute, by funding from the Agency for Healthcare Research and Quality, grant R21 HS013496-02, and by funding from the Health Resources and Services Administration, grant D54 HP00032.

Prior presentation: This research was presented on June 22, 2005, at the Navajo Nation Research Conference in Window Rock, AZ, and on July 21, 2005, at the Primary Care Practice-Based Research Network Conference sponsored by the Agency for Healthcare Research and Quality in Washington, DC.

Conflict of interest: none declared.

Disclaimer: The opinions expressed in this manuscript are those of the authors and do not necessarily reflect those of the Indian Health Service.

Corresponding author: Robert Williams, MD, MPH, University of New Mexico Department of Family and Community Medicine, 2400 Tucker NE, Albuquerque, NM 87131 (E-mail: rlwilliams@salud.unm.edu). tive services delivery. New ideas about the factors that influence delivery of preventive services are emerging. ${ }^{1-8}$ One potential factor influencing a clinician's decision to deliver a preventive service may be a heuristic (ie, an experience-based, problem-solving rule) which allows a clinician to define a particular patient as "high-risk" or "low-risk" for the specific condition targeted by the preventive service. Under the press of competing demands within the brief primary care encounter, the clinician must prioritize among many possible preventive and curative services for use of limited time. ${ }^{8-15}$ Rapid, patient-specific risk estimates during the encounter probably contribute to the clinician's prioritization process. When those risk estimates suggest a patient is at lower need for a specific service, the clinician may be more likely to focus attention on other services and less likely to take time to screen for that specific risk factor or deliver that specific preventive service. ${ }^{11}$

A likely source of information for clinicians' risk estimation for the individual patient is general population-based data; characteristics the patient shares with specific population groups are used to 
assign risk. Such attribution of risk is not only consistent with sound epidemiologic practice but also with literature from cognitive psychology that shows attribution and decision making are often based on visible characteristics. ${ }^{16}$ However, it is not clear that the application of general populationbased risk assessments is always valid for people seen in primary care. In the heuristics and biases literature, the tendency to notice and prioritize information that fits with a pre-existing expectation is known as confirmatory bias. ${ }^{17}$ If indeed the patterns of risk among patients seen in primary care differ from those documented in population groups, the clinician's risk assessment heuristic may suffer from confirmatory bias. In that event, patients' risk status may be misassigned clinically, and preventive services may be inappropriately prioritized among the competing demands.

We conducted an examination of the relationship between general population-based and primary-care based risk information using a survey of tobacco risk behavior in a statewide practice-based research network as a case study of the potential for risk assessment bias. Our research question was to determine the extent to which population-based data accurately reflected the rates of risk factor prevalence seen in primary care. We predicted that general population-based data for this common risk factor would differ importantly from the pattern of prevalence of the same risk factor among patients seen in primary care. If so, this would potentially have important implications for clinician decisionmaking heuristics.

We conducted our study in primary care practices in the southwestern United States, where Hispanics and southwestern Native Americans are reported in population-based data to smoke at lower than average rates. Indeed, the last surgeon general's report on smoking in minority populations lists Hispanic women as one of the least likely groups to smoke. ${ }^{18}$ In the fast-paced environment and competing demands of the brief primary care encounter, clinicians aware of these general populationbased data and seeing someone from these groups may choose to devote the limited encounter time to other pressing topics. Even with the availability of rapid techniques for screening and intervention for tobacco use, such as the 5 A's (ask, assess, advise, assist, arrange), he or she may be less likely to take time to screen for smoking because of an expectation of low risk for smoking.

\section{Methods \\ Study Setting}

We conducted this study in the Research Involving Outpatient Settings Network (RIOS Net), a practice-based research network composed of primary care clinicians serving the state of New Mexico's culturally diverse and often medically underserved populations. RIOS Net's members are predominantly in community health centers, Indian Health Service sites, or academic primary care practices serving low-income communities in New Mexico. We recruited clinicians for participation in the study through a combination of E-mail, fax, and direct telephone or in-person contact. Participating RIOS Net members collected data on all consenting patients 12 years of age and older who presented within a 2 -week period.

\section{Data Collection}

Individual clinicians or their office staff collected data during 2-week periods distributed over calendar years 2004 and 2005 to adjust for seasonal variations in patient presentations. Asking each clinician to collect data on all eligible patients for a 2-week period automatically adjusted for clinician patient volume, removing the need for weighting procedures to correct for differences in patient volume. Clinicians recorded patient demographics and report of current tobacco use (every day or some days), including type of use, duration of use, history of quit attempts, and current interest in quitting. For Native Americans, they also recorded whether the tobacco use was ceremonial, recreational, or both. We based ethnicity on US Census categories and patient self-reporting. Data were analyzed using descriptive statistics, comparing results to published general population-based rates of tobacco use among comparable groups. Four institutional review boards with jurisdiction gave approval to the project.

\section{Sample Size}

The principal research objective was to determine the prevalence rates of tobacco use in patients seen in primary care. For an $\alpha=.05$, to obtain a $95 \%$ confidence interval of length 0.10 or less, a minimum of 246 subjects was needed for an estimate of the proportion of subjects using tobacco, based on an a priori estimate of $20 \%$ tobacco use prevalence. 


\section{Results \\ Clinicians}

A total of 91 RIOS Net clinicians participated in the study. Of the 102 clinicians approached to participate in the study, 9 declined and 2 were unable to complete data collection, for an overall clinician participation rate of $89 \%$. Participating clinicians were $76 \%$ family physicians, $8 \%$ pediatricians, $5 \%$ general internists, and $11 \%$ advanced practice nurses or physician assistants. The clinicians practiced in 32 different clinics throughout the state of New Mexico. The practices were 36\% University associated, $31 \%$ community health centers, 30\% Indian Health Service, and 3\% private practice.

\section{Patients}

Data were collected on 2442 of 2709 eligible patients presenting for care to participating RIOS Net clinicians, for a subject participation rate of $90.2 \%$. The majority $(69 \%)$ of subjects were aged 35 and older, and $63 \%$ were women, reflecting common primary care patient demographic patterns (Table 1). Patients enrolled were $36.6 \%$ Hispanic/Latino, 30\% Native American, 28.1\% nonHispanic white, and $5.3 \%$ black, Asian, or multiethnic. For comparison, the 2000 census for the state of New Mexico reported a population which was $42.1 \%$ Hispanic, $44.7 \%$ non-Hispanic white, and $9.5 \%$ Native American. The participants had the following insurance statuses: $27 \%$ private, 25\% Indian Health Service, 18\% Medicare, $21 \%$ uninsured or indigent program, $5 \%$ Medicaid, and $4 \%$ no charge/other. Statewide insurance status for 2004 was $43 \%$ private, $13 \%$ Medicare, $17 \%$ Medicaid, 21\% uninsured, and $6 \%$ other. ${ }^{19}$

\section{Overall Smoking Rates}

A total of $22.5 \%$ of all subjects smoked cigarettes, and $73.8 \%$ of these people reported daily smoking. Twenty-seven percent of men and $20 \%$ of women smoked cigarettes. The mean rate of use was 9.5 cigarettes per day. An additional $5.5 \%$ of the patients used some other form of tobacco (pipe, cigar, smokeless). Among all tobacco users, the median duration of use was 16 years. Of cigarette smokers, $77.0 \%$ had quit smoking at some time and $63.6 \%$ indicated an interest in quitting. Of those who wanted to quit, $65.1 \%$ were interested in help with quitting.

As expected there were marked differences in smoking prevalence by age. Overall smoking rates
Table 1. Demographic Characteristics of Primary Care Patients Whose Tobacco Use Was Examined $(\mathrm{N}=2442)$

\begin{tabular}{lc}
\hline Demographic & Percentage of Patients \\
\hline Age (years) & \\
$12-17$ & 6.4 \\
$18-24$ & 9.7 \\
$25-34$ & 14.7 \\
$35-44$ & 17.2 \\
$45-54$ & 21.4 \\
$55-64$ & 14.5 \\
65+ & 16.1 \\
Gender & \\
Female & 63.1 \\
Ethnicity & \\
Hispanic & 36.6 \\
American Indian & 30.0 \\
Non-Hispanic white & 28.1 \\
Asian & 2.5 \\
Black & 1.8 \\
Multiethnic & 1.0 \\
Insurance & \\
Private & 27.3 \\
Indian Health Service & 25.0 \\
Medicare & 17.9 \\
No insurance/self pay & 11.1 \\
Indigent Care Program & 10.3 \\
Medicaid & 4.9 \\
Free/no charge & 2.3 \\
Other source & 1.2 \\
\hline
\end{tabular}

were highest among the 18 to 24 age range at $36 \%$ and lowest in the under 18 and over 65 age groups, at $8 \%$ and $10.5 \%$, respectively. Age-specific smoking prevalence also varied markedly by ethnicity. Table 2 summarizes rates by age group and gender. Figures 1 and 2 present rates by age group, gender, and ethnicity for the 3 main ethnic groups in the study. Of note is that the rate of smoking among women overall

Table 2. Smoking Prevalence by Age Group and Gender

\begin{tabular}{ccc}
\hline & \multicolumn{2}{c}{ Percent Smokers $(95 \%$ CI) } \\
\cline { 2 - 3 } & \multicolumn{1}{c}{ Men } & Women \\
\hline Age (years) & & \\
$18-24$ & $50.8(38.6-62.9)$ & $30.0(22.9-37.1)$ \\
$25-34$ & $30.3(21.1-39.4)$ & $24.2(18.8-29.6)$ \\
$35-44$ & $35.1(27.4-42.8)$ & $27.5(22.0-33.0)$ \\
$45-54$ & $29.5(23.3-35.7)$ & $20.8(16.1-25.5)$ \\
$55-64$ & $21.6(14.4-28.8)$ & $17.2(12.1-22.3)$ \\
$65+$ & $14.7(9.0-20.3)$ & $7.7(4.2-11.2)$ \\
\hline
\end{tabular}




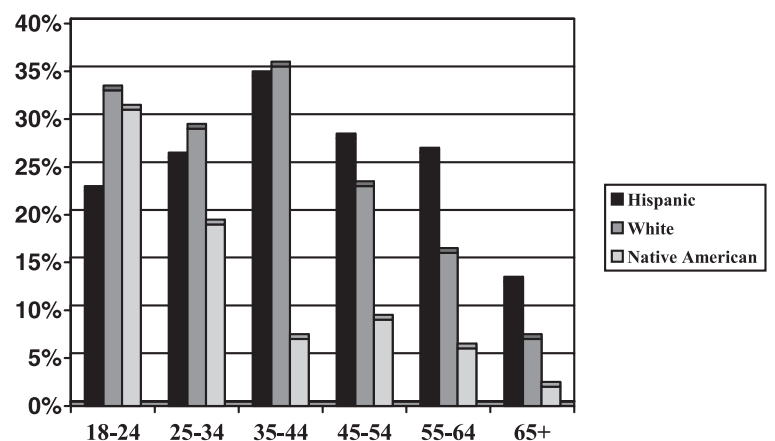

Figure 1. Smoking prevalence for women by ethnicity and age group.

increases throughout the childbearing years, peaking in the late reproductive years. However, as Figure 1 shows, this trend varied by ethnic group.

\section{Comparison of Primary Care and General Population-based Data}

Smoking prevalence varied by ethnic group and gender (Table 3) and differed from general population-based rates as reported by the Behavioral Risk Factor Surveillance System (BRFSS) and the National Health Interview Survey (NHIS). ${ }^{20,21}$ Smoking rates among whites, both men $(33.7 \%)$ and women (21.5\%), were higher than general population-based rates reported for whites in New Mexico by the 2004 BRFSS (19.1\%; the BRFSS does not report gender by race rates for tobacco use). ${ }^{21}$ Similarly, the rates for Hispanic men $(28.3 \%)$ and women $(25.0 \%)$ in these primary care practices were higher than those reported by the 2004 BRFSS for Hispanics in New Mexico $(22.5 \%) .{ }^{21}$ Conversely, the smoking rates for $\mathrm{Na}-$ tive Americans-18.2\% for men and $12.5 \%$ for women-were lower than the national rates reported by the NHIS (BRFSS data are not reported for Native Americans in New Mexico). ${ }^{20}$ However, the smoking rate in the 18 to 24 year old group of Native Americans was approximately double that of rates in other age groups. Figure 3 displays smoking rates by ethnicity and gender among primary care patients in this study compared with general population rates from the NHIS. (Although the rates of smoking among patients seen in primary care practices are best represented by the rates among patients actually seen, we present age-adjusted rates in Table 3 and Figure 3 to permit more direct comparison with general population-based rates.) We also found a difference in daily fre-

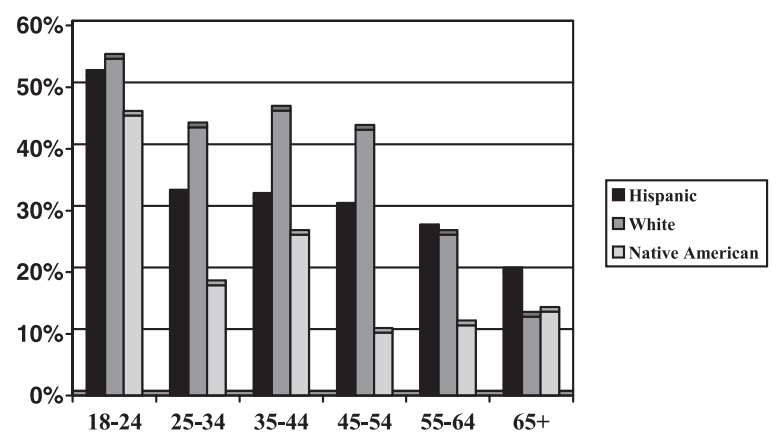

Figure 2. Smoking prevalence for men by ethnicity and age group.

quency of smoking by ethnicity, with $90 \%$ of white and $77 \%$ of Hispanic, but only $38 \%$ of Native American smokers reporting daily tobacco use.

\section{Other Smoking-related Data}

The majority of smokers in this study wanted to quit, including $65 \%$ of female smokers and $62 \%$ of male smokers. Of the 3 major ethnic groups participating in the study, Hispanics reported wanting to quit most frequently (66\%). By gender and ethnicity, Hispanic women reported wanting to quit most frequently (70\%). We also found that $70 \%$ of all smokers had attempted to quit in the past, with the highest rates of previous quit attempts among whites (79\%).

We further examined the issue of ceremonial tobacco use among Native Americans. Twentythree percent of all Native American tobacco users reported that they used tobacco solely for traditional ceremonial or religious reasons, and another $18 \%$ reported tobacco use for both religious and recreational purposes. A further $7 \%$ of Native Americans reported ceremonial tobacco use although they did not classify themselves as tobacco users. We analyzed type of tobacco use by age group and found that recreational use was more frequent with younger persons. For example, 50\% of Native American tobacco users aged 55 to 64 used tobacco only for ceremonial purposes whereas just $6 \%$ of 18 to 24 year old tobacco users reported only ceremonial usage. Native American tobacco users over age 35 were significantly more likely to report "only" ceremonial use than those under 35 years of age $(P=.01)$.

\section{Discussion}

We found important differences in smoking prevalence between our primary care-based sample and 
Table 3. RIOS Net Smoking Prevalence by Gender and Ethnicity

\begin{tabular}{lcccc}
\hline & \multicolumn{4}{c}{ Percent Smokers: Crude and Age Adjusted Rates } \\
\cline { 2 - 5 } Ethnicity & Men (95\% CI) & Men, Age Adjusted & Women (95\% CI) & Women, Age Adjusted \\
\hline Hispanic & $28.0(23.1-33.0)$ & 31.1 & $25.0(21.4-28.5)$ & 26.4 \\
White & $33.7(28.0-39.5)$ & 37.8 & $21.5(17.6-25.4)$ & 24.7 \\
Native American & $18.2(13.6-22.9)$ & 20.6 & $12.5(9.5-15.5)$ & 13.1 \\
\hline
\end{tabular}

general population-based survey results, differences which can only partially be anticipated by the demographic characteristics (eg, low income, ethnicity) of our sample. These differences merit discussion in terms of how they may affect screening for tobacco usage and provision of tobacco cessation services in primary care.

One of the most interesting findings in the current study was that Hispanic women presenting for primary care smoked at much higher rates than might be expected based on population-based surveys $(25.0 \%$ vs $12 \%) .{ }^{20}$ As previously noted, the 1998 Surgeon General's Report on tobacco use in ethnic minorities listed Hispanic women as one of the groups least likely to smoke. ${ }^{18}$ However, because of the ethnic and gender makeup of adult primary care patients in the southwest, Hispanic women constituted the largest proportion $(26 \%)$ of the smokers in our sample. Furthermore, Hispanic women were the group reporting desire to quit most frequently $(70 \%)$ and the second most frequent reporters of wanting help to quit (73\%).

\section{Clinical Implications of Differences between Primary Care and Population-based Risk Estimates}

The discrepancy between general population-based smoking rates and primary care-based rates suggests a possible difficulty in clinician delivery of effective smoking cessation services. Under the pressure of competing demands during the brief primary care encounter, as noted above, it is possible that the provider, using heuristics derived from population-based data, will underestimate the likelihood of a patient's being a smoker and choose to focus on other, non-tobacco related, preventive or curative care activities during the limited time available. The recent literature on preventive services illustrates that in the primary care environment, competing demands capture the time which might have been allocated to tobacco cessation counseling in approximately $50 \%$ to $70 \%$ of eligible visits. ${ }^{11,12}$ Using the example of a Hispanic woman in these practices, the clinician may be less attentive to smoking as a risk for her based on knowledge of population-based rates that identify white males as the most common smokers in New Mexico and Hispanic females as much less common smokers. Under these circumstances and with the press of competing demands in the brief primary care encounter, the clinician may choose to focus on other important topics.

Such a detection bias resulting from reliance on population-based data points to the importance of basing decision-making heuristics on data directly related to the practice: ie, primary care-based data. Ideally, each practice would collect data on a range of preventive and curative risk factors and services relevant to its own patients. Few practices have the

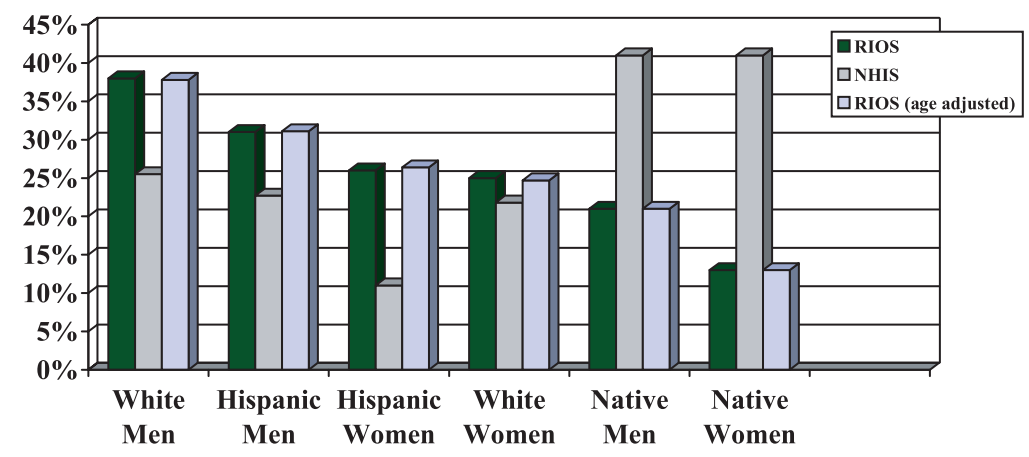

Figure 3. Smoking prevalence by gender and ethnicity: RIOS Net versus 2003 NHIS. 
resources to do this across a broad range of indicators, however. This reality underlines the need for new strategies for providing clinicians with information about their practices and emphasizes the value of participation in practice-based research networks, particularly those that provide practicespecific feedback on care provided.

\section{Other Possible Implications of Differences in Primary Care and Population-based Data}

Another interesting discrepancy between population-based data and primary-care-based data emerged in the area of Native American smoking. An important finding in this primary care group is the apparent pattern of increasing smoking among youth. Our data show that rates of smoking among 18- to 24-year-old Native Americans are markedly higher than the rates in other Native American age groups. Although the cross-sectional design of this study does not permit inference about temporal trends, as Figures 1 and 2 illustrate, the age-based pattern of smoking among Native Americans seen in primary care is unlike that in either of the other ethnic groups. Early knowledge of this pattern of smoking in Native Americans seen in primary care can inform clinical cessation programs in this group.

\section{Limitations}

Our study was conducted in primary care practices that differ from those in the general population. Although the specific rates of smoking that we found may not generalize to other segments of the population, the finding of differences between practice-based and general population-based rates is more likely to be generalizable. Our data rely on self-reporting of smoking status, which has been determined in other circumstances to not fully match biochemical measures of smoking. ${ }^{22}$ Nevertheless, the use of self-reporting does match the reality of primary care practice, reflecting the information on patient smoking available to clinicians. We did not collect extensive data on socioeconomic status (SES), a well-known determinant of smoking status. It is possible that our sample contained disproportionate numbers of people with low incomes and that our observed rates of smoking in primary care patients were reflective of this. However, our findings on lack of health insurance, which may be considered a broad proxy for SES, show that our sample had a comparable rate of uninsurance $(21 \%)$ as the rate of uninsurance (22\%) statewide. This suggests that SES did not greatly influence the differences we observed between population-based and primary care-based smoking rates. Finally, our study was not designed to explore or confirm the extent to which clinician prior expectations about individual patient risk, based on population group characteristics, may influence clinician decisions to prioritize preventive care for that risk in the brief encounter. As mentioned, this will be an important topic for future research.

\section{Conclusions}

Our case study examining tobacco use found important differences between the general population-based distribution of a key health risk factor and the distribution of that risk among patients seen in a large group of primary care practices. Our study suggests that the prevalence patterns of smokers seen by primary care providers are likely to be quite different from those of smokers in general population-based samples. In the context of multiple competing demands within the brief primary care encounter, these differences could have important implications for delivery of an effective smoking cessation message. Further research is necessary to determine whether practice-specific information can help change clinician expectations and behavior with regard to targeting of preventive care in the brief encounter. However, we believe that these data emphasize the broader importance of, and need for, primary care-based data and practice-based research to both understand and improve primary care practice. Primary care practice is sufficiently distinct that reliance on data collected in other contexts can both mislead and make improvements more difficult.

\section{References}

1. Miller WL, Crabtree BF, McDaniel R, Stange KC. Understanding change in primary care practice using complexity theory. J Fam Pract 1998;46:369-76.

2. Miller WL, McDaniel RR Jr, Crabtree BF, Stange KC. Practice jazz: understanding variation in family practices using complexity science. J Fam Pract 2001;50:872-8.

3. Crabtree BF, Miller WL, Stange KC. Understanding practice from the ground up. J Fam Pract 2001; 50:881-7.

4. Stroebel CK, McDaniel RR Jr, Crabtree BF, Miller WL, Nutting PA, Stange KC. How complexity sci- 
ence can inform a reflective process for improvement in primary care practices. Jt Comm J Qual Patient Saf 2005;31:438-46.

5. Litaker D, Tomolo A, Liberatore V, Stange KC, Aron D. Using complexity theory to build interventions that improve health care delivery in primary care. J Gen Intern Med 2006;21:S30-4.

6. Matlow AG, Wright JG, Zimmerman B, Thomson $\mathrm{K}$, Valente M. How can the principles of complexity science be applied to improve the coordination of care for complex pediatric patients? Qual Saf Health Care 2006;15:85-8.

7. Sussman A, Williams RL, Leverence R, Gloyd PW, Crabtree BF. The art and complexity of primary care clinicians' preventive counseling decisions: obesity as a case study. Ann Fam Med 2006;4:327-33.

8. Crabtree BF, Miller WL, Aita VA, Flocke SA, Stange KC. Primary care practice organization and preventive services delivery: a qualitative analysis. J Fam Pract 1998;46:403-9.

9. Jaen CR, Stange KC, Nutting PA. Competing demands of primary care: a model for the delivery of clinical preventive services. J Fam Pract 1994;38: $166-71$.

10. Yarnall KS, Pollak KI, Ostbye T, Krause KM, Michener JL. Primary care: is there enough time for prevention? Am J Public Health 2003;93:635-641.

11. Jaen CR, Stange KC, Tumiel LM, Nutting P. Missed opportunities for prevention: smoking cessation counseling and the competing demands of practice. J Fam Pract 1997;45:348-54.

12. Jaen CR, McIlvain H, Pol L, Phillips RL Jr, Flocke $\mathrm{S}$, Crabtree BF. Tailoring tobacco counseling to the competing demands in the clinical encounter. J Fam Pract 2001;50:859-63.

13. Nutting PA, Baier M, Werner JJ, Cutter G, Conry C, Stewart L. Competing demands in the office visit: what influences mammography recommendations? J Am Board Fam Pract 2001;14:352-61.

14. Rost K, Nutting P, Smith J, Coyne JC, CooperPatrick L, Rubenstein L. The role of competing demands in the treatment provided primary care patients with major depression. Arch Fam Med 2000; 9:150-4.

15. Chernof BA, Sherman SE, Lanto AB, Lee ML, Yano EM, Rubenstein LV. Health habit counseling amidst competing demands: effects of patient health habits and visit characteristics. Med Care 1999;37:738-47.

16. Burgess DJ, Fu SS, van Ryn M. Why do providers contribute to disparities and what can be done about it? J Gen Intern Med 2004 Nov;19:1154-9.

17. Kahneman D, Slovic P, Tversky A, editors. Judgement under uncertainty: heuristics and biases. Cambridge: Cambridge University Press; 1982.

18. Tobacco use among US racial/ethnic minority groups-African American, American Indians and Alaska Natives, Asian Americans and Pacific Islanders, and Hispanics: a report of the surgeon general. Atlanta (GA): US Department of Health and Human Services, Centers for Disease Control and Prevention, National Center for Chronic Disease Prevention and Health Promotion, Office on Smoking and Health; 1998.

19. The Kaiser Family Foundation statehealthfacts.org [database on the Internet]. Data source: Census Bureau's March 2005 and 2006 Current Population Survey (CPS: Annual Social and Economic Supplements). Urban Institute and Kaiser Commission on Medicaid and the Uninsured. [cited 18 October 2006]. Available from: http://www.statehealthfacts.org.

20. Lethbridge-Çejku M, Vickerie J. Summary health statistics for US adults: National Health Interview Survey, 2003. National Center for Health Statistics. Vital Health Stat 2005;10(225).

21. Prevalence Data, New Mexico-2004, Tobacco Use, Behavioral Risk Factor Surveillance System [database on the Internet]. Atlanta (GA): National Center for Chronic Disease Prevention and Health Promotion. [cited 7 October 2006]. Available from: http:// apps.nccd.cdc.gov/brfss/race.asp?cat $=\mathrm{TU} \& \mathrm{yr}=$ 2004\&qkey $=4396 \&$ state $=$ NM.

22. Perez-Stable EJ, Marin G, Marin BV, Benowtiz NL. Misclassification of smoking status by self-reported cigarette consumption. Am Rev Respir Dis 1992; 145:53-7. 